\title{
Theoretical Analysis of Optical Losses in CdS/CdTe Solar Cells
}

\author{
V.Ya. Roshko, L.A. Kosyachenko and E.V. Grushko \\ Optoelectronics Department, Chernivtsi National University, Kotsyubinsky Str. 2, 58012 Chernivtsi, Ukraine \\ Based on the known refractive index and extinction coefficient, calculations of optical losses in glass/ \\ transparent conducting oxide/CdS/CdTe solar cells have been carried out taking into account reflections at the \\ interfaces and absorption in the transparent conducting oxide (indium tin oxide or $\mathrm{SnO}_{2}: \mathrm{F}$ ) and CdS layers. It \\ has been shown that the losses caused by reflections at the interfaces result in lowering the short-circuit current \\ by $\approx 9 \%$ whereas absorption in the transparent conducting oxide and CdS layers with the typical thicknesses lead \\ to losses of $15-16 \%$ for glass $/ \mathrm{SnO}_{2} / \mathrm{CdS} / \mathrm{CdTe}$, and $22-24 \%$ for glass/indium tin oxide/CdS/CdTe solar cells.
}

PACS: 84.60.Jt, 85.60.Dw, 73.61.Ga

\section{Introduction}

Since the beginning of the last decade mass production of solar modules based on thin-film CdS/CdTe has been started [1]. Today the challenge facing the researchers and technologists is how to increase the efficiency of CdS/ CdTe modules from the current efficiency of $10-11 \%$ and to decrease the gap between actual efficiency and the theoretical limit of $28-30 \%$. The main causes of efficiency loss in $\mathrm{CdTe} / \mathrm{CdS}$ solar cells are optical, electrical and recombination losses, a topic of study discussed in a substantial amount of papers. The present study focuses on optical losses due to absorption and reflection at the interfaces in $\mathrm{CdTe} / \mathrm{CdS}$ solar cells. Calculations have been carried out based on the optical constants of materials used, the refractive index and extinction coefficient. It seems that the results of these calculations are interesting from a scientific and practical point of view.

\section{Calculations of reflection and absorption losses}

Before reaching the photoelectrically active $\mathrm{CdTe}$ absorber layer, solar radiation penetrates the glass plate, a layer of transparent conducting oxide (TCO) and CdS window layer. Obviously, this is accompanied by optical losses upon reflection from the following interfaces: air-glass (reflection coefficient $\left.R_{12}\right)$, glass-TCO $\left(R_{23}\right)$, TCO-CdS $\left(R_{34}\right)$, and CdS-CdTe $\left(R_{45}\right)$, and absorption in TCO and CdS. According to the Fresnel equations, when the light is at near-normal incidence, the reflection coefficient from the interface between two contacting materials is determined by their refractive indices $n_{1}$ and $n_{2}$, i.e. $R=\left(n_{1}-n_{2}\right)^{2} /\left(n_{1}+n_{2}\right)^{2}$. In the case of electrically conductive materials, refractive index contains an imaginary part and is written as $n^{*}=n-\mathrm{i} \kappa$, where $n$ is the refractive index, and $\kappa$ is the extinction coefficient. The expression for reflection coefficient from the interface has the form [2]:

$$
R=\frac{\left|n_{1}^{*}-n_{2}^{*}\right|^{2}}{\left|n_{1}^{*}+n_{2}^{*}\right|^{2}}=\frac{\left(n_{1}-n_{2}\right)^{2}+\left(\kappa_{1}-\kappa_{2}\right)^{2}}{\left(n_{1}+n_{2}\right)^{2}+\left(\kappa_{1}+\kappa_{2}\right)^{2}} .
$$

At the air-glass interface we will find the reflection coefficient $R_{12}$ taking $n_{1}=1$, and $\kappa_{1}=0$ for air. For the glass, it is possible to take $\kappa_{2}=0$ and $n_{1}$ is given by the Zelmeer formula applied for quartz $\left(\mathrm{SiO}_{2}\right)$ [3].

To find the reflection coefficients at the interfaces: glass-TCO, TCO-CdS, and CdS-CdTe it is necessary to know the values of the refractive index and extinction coefficient of TCO, CdS and CdTe in the spectral range 300-850 $\mathrm{nm}$. In the discussion below we consider the two most common structures of $\mathrm{CdTe} / \mathrm{CdS}$ solar cell in which indium tin oxide (ITO) and F-doped tin oxide $\left(\mathrm{SnO}_{2}: \mathrm{F}\right)$ are used as TCO. The spectral dependences of $n$ and $\kappa$ for ITO (typically $90 \% \mathrm{In}_{2} \mathrm{O}_{3}, 10 \% \mathrm{SnO}_{2}$ ) and $\mathrm{SnO}_{2}$ taken from Refs. [4, 5] are presented in Fig. 1, together with data on CdS [6] and CdTe [7].

Figure 2 shows the spectral dependence of the reflection coefficients $R(\lambda)$ at the interfaces calculated by substituting $n$ and $\kappa$ from Fig. 1 into Eq. (1). Emphasis is given on obtaining low reflection coefficients (less than 0.05-0.06). The explanation for this is a relatively small difference between the optical constants of contacting materials.

For reflection losses at all interfaces before the solar radiation reaches the CdTe absorber layer, the transmission $T(\lambda)$ can be calculated by the formula

$$
T(\lambda)=\left(1-R_{12}\right)\left(1-R_{23}\right)\left(1-R_{34}\right)\left(1-R_{45}\right) .
$$

It follows from Fig. 3 that the total reflection losses at the interfaces: air-glass, glass-ITO, ITO-CdS, and CdSCdTe in the thin-film CdS/CdTe solar cell is about $9 \%$ in 

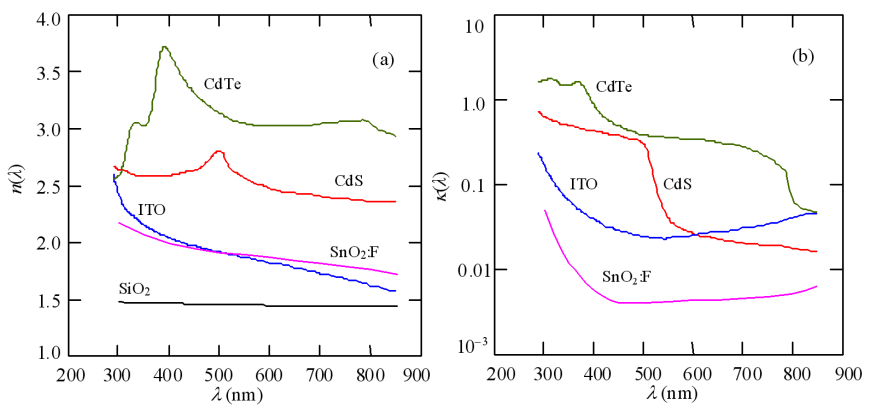

Fig. 1. Refractive index (a) and extinction coefficient (b) of $\mathrm{SiO}_{2}$, ITO, $\mathrm{SnO}_{2}: \mathrm{F}, \mathrm{CdS}$ and CdTe as a function of wavelength.

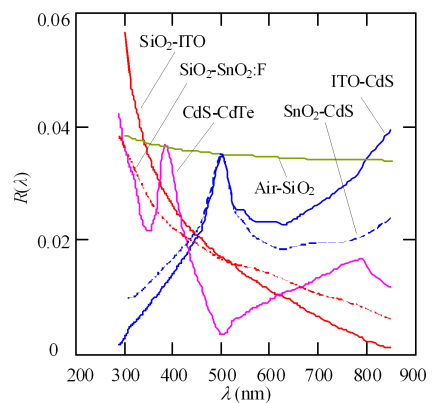

Fig. 2. Reflection coefficients $R$ for the interfaces: airglass, glass-ITO, glass- $\mathrm{SnO}_{2}, \mathrm{SnO}_{2}-\mathrm{CdS}, \mathrm{CdS}-\mathrm{CdTe}$.

the entire spectral range $300-850 \mathrm{~nm}$. This implies that the reflection losses in the case of thin-film $\mathrm{CdS} / \mathrm{CdTe}$ structure are not as critical as in the case of a silicon solar cell.

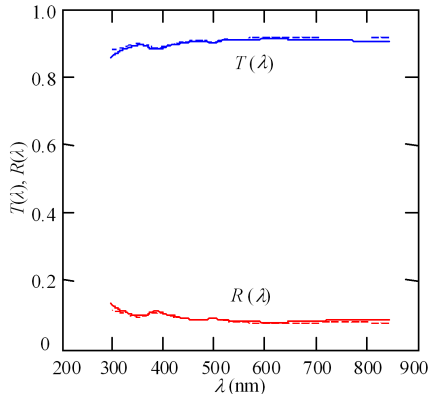

Fig. 3. Calculated transmittance $T(\lambda)$ and reflectance $R(\lambda)$ of the glass/ITO/CdS (solid line) and glass $/ \mathrm{SnO}_{2} /$ CdS (dashed line) structures with account made only for reflections by all interfaces.

Equation (2) does not account for multiple reflections in the glass plate, ITO and CdS layers, which is quite acceptable because of the small difference of reflection coefficients at the interfaces (Fig. 2).

Let us pass to the absorption losses. The data for the extinction coefficient $\kappa(\lambda)$ shown in Fig. 1b allows to find the absorption coefficient as $\alpha(\lambda)=2 \omega \kappa / c$, where $\omega$ is the angular frequency, and $c$ is the speed of light in free space. Knowing $\alpha(\lambda)$ for the CdS and ITO $\left(\mathrm{SnO}_{2}\right)$, the absorption in these layers can be taken into account by incorporating in Eq. (2) the addition factors $\exp \left(-\alpha_{\mathrm{CdS}}(\lambda) d_{\mathrm{CdS}}\right)$, $\exp \left(-\alpha_{\mathrm{ITO}}(\lambda) d_{\mathrm{ITO}}\right), \exp \left(-\alpha_{\mathrm{SnO}}(\lambda) d_{\mathrm{SnO}}\right)$ :

$$
\begin{aligned}
& T(\lambda)=\left(1-R_{12}\right)\left(1-R_{23}\right)\left(1-R_{34}\right)\left(1-R_{45}\right) \\
& \quad \times \exp \left(-\alpha_{\mathrm{ITO}} d_{\mathrm{ITO}}\right) \exp \left(-\alpha_{\mathrm{CdS}} d_{\mathrm{CdS}}\right), \\
& T(\lambda)=\left(1-R_{12}\right)\left(1-R_{23}\right)\left(1-R_{34}\right)\left(1-R_{45}\right) \\
& \quad \times \exp \left(-\alpha_{\mathrm{SnO}} d_{\mathrm{SnO}}\right) \exp \left(-\alpha_{\mathrm{CdS}} d_{\mathrm{CdS}}\right),
\end{aligned}
$$$$
\text { or }
$$

where $d_{\mathrm{ITO}}, d_{\mathrm{SnO}}$ and $d_{\mathrm{CdS}}$ are the thicknesses of the ITO, $\mathrm{SnO}_{2}: \mathrm{F}$ and $\mathrm{CdS}$ layers, respectively.

Calculation of short circuit current can quantitatively account for the effect of optical losses in the entire spectral range. We will calculate the short-circuit current density $J_{\mathrm{sc}}$ for AM1.5 total solar radiation using the Table ISO 9845-1:1992 [8].

If $\Phi_{i}$ is the spectral radiation power density, $h \nu$ is the photon energy and the spectral density of the incident photon flux is $\Phi_{i} / h \nu_{i}$, then we can write $J_{\mathrm{sc}}$ as

$$
J_{\mathrm{sc}}=q \sum_{i} T(\lambda) \frac{\Phi_{i}(\lambda)}{h v_{i}} \Delta \lambda,
$$

where $\Delta \lambda_{i}$ is the wavelength range between the neighboring values of $\lambda_{i}$ (the photon energy $h \nu_{i}$ ) in the table and the summation is over the spectral range $\lambda=300 \mathrm{~nm}$ to $\lambda=\lambda_{\mathrm{g}}=h c / E_{\mathrm{g}} \approx 840 \mathrm{~nm}$.

Figure 4 shows the dependence of short circuit current $J_{\mathrm{sc}}$ on the thickness of ITO and $\mathrm{SnO}_{2}: \mathrm{F}$ calculated for different thickness of CdS with the assumption that the efficiency of photoelectric conversion in the CdTe layer is equal to $100 \%$.

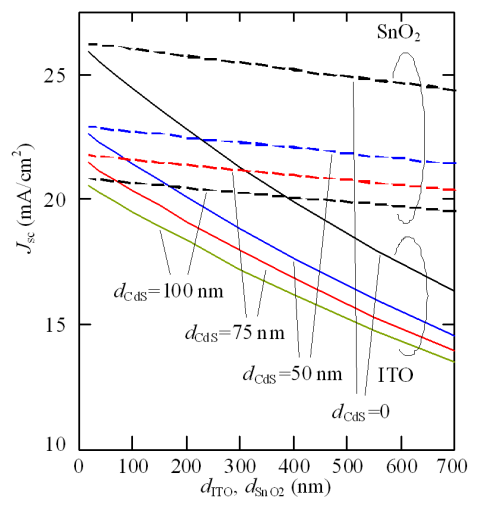

Fig. 4. Effect of optical losses on short-circuit current depending on the thickness of ITO and $\mathrm{SnO}_{2}: \mathrm{F}$ layer calculated for different thicknesses of CdS layer.

As can be seen from Fig. 4, optical losses caused by TCO layer with thickness of $200 \mathrm{~nm}$ are about $20 \%$ and $10 \%$ for ITO and $\mathrm{SnO}_{2}: \mathrm{F}$, respectively. When the thickness of the TCO layer is close to 0 , the optical losses are caused only by reflection and become equal to 8.5 
and $8.8 \%$ for the $\mathrm{SnO}_{2}: \mathrm{F}$ and ITO layers, respectively. Thicker ITO layers of the order of 500-700 nm leads to unacceptably high optical losses $(\approx 50 \%)$, while using $\mathrm{SnO}_{2}: \mathrm{F}$ the losses are reduced to a few percentage points only. In both cases, the presence of CdS layer, even when its thickness is $50 \mathrm{~nm}$ leads to further increase in the optical losses by approximately $10 \%$ and for a thickness of $100 \mathrm{~nm}$ the loss increases by another $7-8 \%$. It should be borne in mind that the thickness of CdS equal to 50 $60 \mathrm{~nm}$ is close to the minimum possible value with the current technology used in the manufacturing of CdS/ CdTe solar cells. As is evident from these data, a short-circuit current density above $\approx 25 \mathrm{~mA} / \mathrm{cm}^{2}$, is practically impossible with the use of pure CdS as the window layer.

\section{Conclusions}

The results of the calculations reveal the specific causes of the optical losses in the $\mathrm{CdS} / \mathrm{CdTe}$ solar cell and the possibilities to reduce them. (i) Reflection losses are about $8 \%$ over a wide spectral range, and only in the region $\lambda<400 \mathrm{~nm}$ increase to $10-11 \%$. Certainly, reflection losses can be reduced by $\approx 4 \%$ using antireflection coating on the front surface of the glass. (ii) Due to absorption in the ITO layer of $200 \mathrm{~nm}$ thickness, the short-circuit current decreases by $12 \%$, however, the decrease can be $25-26 \%$ when the thickness is $500 \mathrm{~nm}$. In contrast, absorption in the $\mathrm{SnO}_{2}: \mathrm{F}$ layer is much smaller, causing only a decrease in short circuit current of about $2 \%$ at a thickness of $200 \mathrm{~nm}$, and $4-5 \%$ at a thickness of $500 \mathrm{~nm}$. (iii) Absorption loss in CdS cannot be avoided by reducing thickness alone, since it is related to inter- band optical transitions. Even at a thickness of $50 \mathrm{~nm}$, decrease in the short-circuit current, caused by absorption in the CdS layer, is about $10 \%$ and further increase of $5-6 \%$ occurs at a thickness of $100 \mathrm{~nm}$.

\section{Acknowledgments}

This study was supported by Fundamental Researches State Fund, Ukraine within the Agreement $\Phi 40.7 / 014$.

\section{References}

[1] http://www.firstsolar.com/en/modules.php .

[2] T.S. Moss, G.J. Burrel, D. Ellis, Semiconductor Opto-Electronics, Butterworth Publ., Belfast 1973.

[3] S.O. Kasap, Optoelectronics and Photonics: Principles and Practice, Prentice Hall, New Jersey 2000, Ch. 1.

[4] E. Fred Schubert, Educational Resources. Refractive Index and Extinction Coefficient of Materials, Rensselaer Polytechnic Institute, New York 2007.

[5] A. Compaan, R. Collins, V. Karpov, D. Giolando, Annual Technical Report, June 1, 2006 to May 31, 2007. Contract No. RXL-5-44205-01. The University of Toledo, Toledo, OH 43606.

[6] S. Ninomiya, Sadao Adachi, J. Appl. Phys. 78, 1183 (1995).

[7] T. Toshifumi, S. Adachi, H. Nakanishi, K. Ohtsuka, Jpn. J. Appl. Phys. 32, 3496 (1993).

[8] Reference Solar Spectral Irradiance at the Ground at Different Receiving Conditions, Standard of International Organization for Standardization ISO 9845-1: 1992. 